\title{
Perfil epidemiológico de las enfermeras Adultas Mayores jubiladas de Rio Gallegos. Percepción de Salud y su relación con la polifarmacia
}

\section{Epidemiological profile of retired older-adult nurses from Rio Gallegos. Perception of Health and its relationship with polypharmacy}

\author{
Pablo Crotti, Sara Ojeda, María Laura Ivanissevich \\ pablocrotti@hotmail.com,sojeda@uarg.unpa.edu.ar,mivanissevich@uarg.unpa.edu.ar \\ ISISC - UARG \\ Universidad Nacional de la Patagonia Austral \\ Santa Cruz - Argentina
}

Recibido: 11/02/2019. Aceptado: 05/11/2020

\begin{abstract}
RESUMEN
El estudio de la auto-percepción de la salud que tienen las enfermeras jubiladas Adultas Mayores (AM) de Río Gallegos reveló que el perfil epidemiológico en referencia a las enfermedades crónicas, es coincidente con las estadísticas nacionales de salud; más del cincuenta por ciento indicó padecer alteraciones cardiovasculares y osteo-musculares y aun así perciben su salud como muy buena. Han mostrado un estilo saludable en el manejo y administración de los fármacos en relación a enfermedades crónicas y tratamiento médico, y la polifarmacia no se ha revelado como un problema en esta población.
\end{abstract}

Palabras clave: enfermeras jubiladas; adulto mayor; percepción de salud; polifarmacia; Rio Gallegos

\begin{abstract}
This study presents the self-perception of the health of retired older-adult nurses of Río Gallegos. Results revealed that the epidemiological profile in reference to chronic diseases of nurses coincides with national health statistics. More than 50\% indicated that they suffer from cardiovascular and osteomuscular alterations, although they perceive that they have a very good health. They present a healthy style in the management and administration of drugs in relation to chronic diseases and medical treatment, which reveals that polypharmacy is not a problem in this population.
\end{abstract}

Keywords: nursing; retirement; older-adult; perception of health; polypharmacy; Rio Gallegos

\section{INTRODUCCIÓN}

Argentina es el tercer país más envejecido de la región latinoamericana siendo superado por Cuba y Uruguay, la población argentina es cada vez más vieja; la pirámide poblacional del 2010 muestra el estrechamiento de la base, es decir de las edades de 0 a 4 años y el ensanchamiento en la cúspide de la pirámide -más de 65 años- y la provincia de Santa Cruz también está inmersa en esa dinámica (INDEC, 2010). El envejecimiento de la población es 
un fenómeno mundial con implicancias políticas socio-económicas que conlleva múltiples necesidades de servicios de salud, de previsión social, de trabajo, vivienda y recreación entre otras. Plantea nuevos desafíos generando demandas específicas y complejas siendo una de ellas la de personal calificado para brindar el cuidado a los adultos mayores (CEPAL, 2010).

Los cambios fisiológicos naturales del envejecimiento llevan consigo un incremento de las probabilidades de padecer enfermedades crónicas y discapacidad, con el consiguiente aumento en el consumo de fármacos. Hoy las enfermedades crónicas prevalecen en forma epidémica y su consolidación se produjo por la generalización de estilos de vida no saludables. En contraparte un actual sistema de salud deteriorado con desventajosas políticas sociales y de salud para la población AM marca para ellos un escenario de total vulnerabilidad.

La Atención primaria de la salud (APS) adquiere especial relevancia al momento de brindar el cuidado, ya que además de intervenir en forma temprana, permite evaluar las necesidades de apoyo y de aprendizaje de las personas Adultas Mayores (AM) con el fin de generar estrategias para la incorporación de estilos de vida saludable que minimicen los factores de riesgos para las enfermedades crónicas y por ende la polifarmacia, garantizando la seguridad en el uso racional de los fármacos (Peláez, 2015).

La Organización Mundial de la Salud (OMS, 2000) ha definido a los trabajadores de la salud como profesionales altamente estresados. Particularmente los profesionales de enfermería presentan tendencia a padecer el síndrome de estrés asistencial (Novoa Gómez, 2005) mostrando cansancio emocional y sentimientos de impotencia, entre otras señales que pueden conducir a desarrollar enfermedades crónicas, vida sedentaria y/o un abuso en el consumo de fármacos.

La auto-percepción se constituye como un indicador importante para conocer el estado de salud de los AM, se incorpora a mitad del siglo XX y desde entonces es ampliamente aceptado como una medida confiable del estado de salud general. Es una información subjetiva que integra factores biológicos, mentales, sociales y funcionales del individuo. El proyecto de investigación "La población enfermera jubilada adulta mayor de Río Gallegos; percepción de su estado de salud", incluyó como categorías de análisis la morbilidad crónica y la polifarmacia con el fin de relacionarlas a la auto-percepción de la salud que tienen las personas jubiladas AM que supieron desarrollarse como trabajadores de la enfermería. Debido a los escasos estudios zonales descriptivos que se registran sobre la calidad de vida de este colectivo tan particular, surge como muy interesante el profundizar el conocimiento sobre el mismo.

\section{DESARROLLO}

\subsection{Envejecimiento y Salud}

Mella y otros (2004) expresan que hacia el 2025 por los grandes avances sociales técnicos y científicos habría una población de 1000 millones de adultos mayores y que por primera vez será más numerosa que los jóvenes. Datos del INDEC, Argentina (2010) ubican a Santa Cruz con una población de 22.143 AM, representando el $8.03 \%$ de la población total.

La provincia de Santa Cruz al igual que Argentina tiene aspectos poblacionales muy peculiares debido a que se conformó y condicionó por movimientos migratorios, de países limítrofes y migraciones internas, situación ésta que incide en la población AM, no sólo en lo 
que al aspecto demográfico se refiere sino a las implicancias y requerimientos de atención de salud que ello conlleva.

Este patrón de cambio en la estructura poblacional que se inicia con la disminución de la mortalidad materno-infantil, el aumento en la esperanza de vida, un posterior descenso de la natalidad y la permanencia de los grupos familiares que no vuelven otra vez a inmigrar, genera un envejecimiento de la población con las consiguientes variaciones de los problemas de salud y prioridades de atención que desafían la capacidad de respuesta de los sistemas de salud y de la población en su conjunto.

En cada momento histórico se han expresado trascendentales variaciones en la concepción de la salud-enfermedad; siendo los cambios más notables en la segunda mitad del siglo XX. La Organización Mundial de la Salud (OMS) ha propiciado un rol importantísimo como ente aglutinador de las últimas tendencias en el campo de la salud, pudiendo decirse que en la actualidad suele aceptarse un concepto de salud ecológico, sistémico, dinámico y positivo, resultante de la interacción y adaptación del hombre a su medio físico-social y es por ello que hoy hablamos de "La salud y su complejidad". Cuando se hace mención a la salud, entonces, es importante entenderla como un proceso dinámico inserto en la sociedad de la que forma parte y que constituye un indicador en sí mismo, por ser el encuentro entre la naturaleza humana y su organización social, y que además da cuenta del bienestar y de la calidad de vida de una comunidad.

En tiempo en que las miradas y los esfuerzos económicos se orientan a la biología humana y las organizaciones de los sistemas de salud, se destaca el informe Lalonde (Canadá 1974), que marcó un punto de inflexión y ofreció una herramienta de cambio en la comprensión de la salud basada en el concepto de campo de la salud jerarquizando el ambiente y los estilos de vida, entre los determinantes de salud. Si bien es claro que la biología humana, inherente a lo genético domina la susceptibilidad individual de enfermar también lo es que el entorno y el estilo de vida dominan la incidencia y la prevalencia de las enfermedades. El campo de la salud refleja y aumenta los cambios como un zoom, por ello es necesario comprender el proceso si pretendemos anticiparnos a los hechos.

Esta realidad de vivir más años implica un desafío personal de adaptación a los distintos cambios propios de la edad como lo son los biológicos y funcionales y por otra parte los cambios psicosociales que esta etapa trae, como menor participación activa en la sociedad que suele estar asociada a la jubilación. Es en esta etapa del ciclo vital donde es aplicable para las enfermeras, la teoría de Callista Roy que define salud como un proceso de adaptación en el mantenimiento de la integridad fisiológica, psicológica y social donde el "hombre" es un complejo sistema biológico que trata de adaptarse a los cuatro aspectos de la vida: fisiológico, autoimagen, dominio del rol e interdependencia. Aspectos que atraviesan todos los cambios producidos en el envejecimiento, en los que el AM interacciona con el entorno ejerciendo y recibiendo influencias que intervienen en su autoimagen y el dominio del papel social.

Las enfermeras basan sus cuidados en diversas teorías y básicamente todas ellas -en la actualidad- están orientadas al cuidado desde una visión holística que toma en cuenta al hombre en todas sus dimensiones y en estrecha vinculación con su medio, por lo que cabría suponer que la práctica de la teoría de la adaptación durante todo el ejercicio de la profesión, sería aplicable para ellas mismas al momento de acceder a la etapa de AM. 
El envejecimiento como proceso continuo, heterogéneo y universal no es un proceso exclusivamente biológico y psicológico sino también sociocultural. Es durante ese proceso que el AM se asume y se construye en el nuevo rol, con él se integra y se reinserta en la comunidad marcando su nuevo lugar. Coexisten una serie de términos que aluden a esta franja etaria, tales como tercera edad, AM, personas de edad o "jubilados", surgiendo esta última denominación en la década de los sesenta cuando se instaura la jubilación universal en Francia y que significó un cambio profundo en su rol social. La jubilación cobra importancia en la medida que reconoce factores que distinguen a esta población y que por su condición de mayoritaria se ha convertido en una designación usual para las personas de edad avanzada y que determina ciertos estilos de vida y de relación con la sociedad y con el Estado. Por otra parte vale destacar que cada sociedad construye su propia concepción acerca de lo que significan las edades del ser humano y entre ellas la vejez, por lo que, tanto la jubilación como la noción de la edad se encuentran ligadas a contextos socio histórico concretos.

Stoller y Gibson (1994) refieren que...

" más importante que la propia influencia social y biológica son las relaciones que se dan entre estas fuerzas, los cambios no se entienden si uno no los considera a la luz de los acontecimientos históricos, experiencias individuales, fuerzas sociales y culturales que definen los tiempos".

Para el caso de la población de estudio, enfermeras jubiladas AM en Río Gallegos, cabe contextualizar que para acceder a la jubilación, el sistema previsional de la Provincia de Santa Cruz requiere 28 años de aporte continuo para las mujeres y 30 años para los hombres sin límite de edad y si es con aportes a otras cajas de jubilación un minino de 50 años para las mujeres y 54 para los hombres y más de veinte años de aporte a la provincia; lo que significa que en un gran porcentaje esta población se encuentra jubilada aun antes de alcanzar los 60 años. Para el sistema previsional nacional argentino el requisito para acceder a la jubilación es de 30 años de aporte y 60 años de edad para las mujeres y 65 para los hombres.

\subsection{Envejecimiento y Contexto epidemiológico}

El envejecimiento de la población, en nuestra región, se produce a un ritmo más acelerado que el crecimiento económico y social representando un desafío para las políticas socio-sanitarias; lo ideal es que la comunidad pueda establecer una relación reciproca entre los niveles de salud y el grado de desarrollo económico, de tal forma de promover la igualdad de oportunidades a toda este colectivo poblacional. Pensamiento que remonta al año 1949 en el que el Dr. Ramón Carrillo con una visión social de la salud muy amplia explicitaba que "...no puede haber política sanitaria sin política social..."

En este siglo XXI de marcada transición demográfica, el modelo de Julio Frenk y col. (Barragán, 2007) ya en el año 1981 planteaba las variaciones en las condiciones de salud que se evidenciaban a través de dos componentes: la transición en las condiciones de salud que definen el perfil epidemiológico y la transición de la atención sanitaria que expresa la respuesta social o perfil asistencial. Esta transición epidemiológica muestra un giro de las enfermedades infecciosas hacia las enfermedades crónicas por un mejoramiento general de las condiciones sanitarias ambientales. Hoy las enfermedades crónicas prevalecen en forma epidémica y su consolidación no se produjo por el control de las enfermedades infecciosas sino por la generalización de estilos de vida no saludables. En contraparte, un actual sistema de salud deteriorado con desventajosas políticas sociales para la población AM marca un escenario de marcada vulnerabilidad para ellos. 
Datos del INDEC (2010) dan cuenta del perfil epidemiológico de los AM en la Argentina y muestra que las enfermedades más frecuentes son las siguientes: disfunciones cardiovasculares, cáncer, accidente cerebro-vascular, diabetes, infecciones, artrosis y osteoporosis, trastornos neuro-psiquiátricos y debe tenerse especial consideración a los Gigantes de la Geriatría; inestabilidad y caídas, incontinencia urinaria, inmovilidad, deterioro cognitivo y polifarmacia. Se suman las alteraciones fisiológicas propias del envejecimiento como la disminución auditiva y visual que no constituyen enfermedades en sí, pero influyen en la funcionalidad y grados de independencia del AM. En la población en estudio se encontró que solo un $10 \%$ refirió disfunción auditiva y un $60 \%$ disfunción visual.

\section{Disfunción Auditiva}

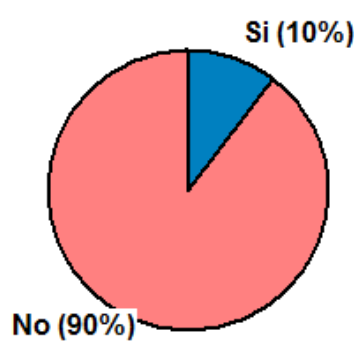

Disfunción Visual

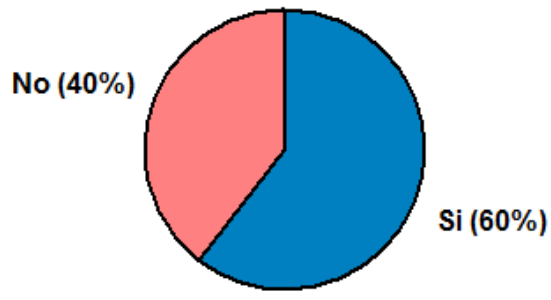

Fuente: Proyecto Investigación UARG.UNPA

González García G (2004), indican que las enfermedades crónicas prevalentes varían según la edad, para el caso de la hipertensión arterial representa un $60 \%$ en mayores de 60 años y para la diabetes tiene una prevalencia del $20 \%$ en esta población. También se destaca la relación entre ambas enfermedades con otras enfermedades crónicas como la obesidad y enfermedades coronarias. Por otra parte Fasio, A. Roque, M (2008), expresan que a partir de los 75-80 años se presentan la mayoría de los trastornos biológicos, pero solo el $12 \%$ de los mayores de 80 años presenta algún grado de discapacidad biológica o mental.

En la población AM de enfermeras jubiladas de Rio Gallegos, con un porcentaje del $85 \%$ de mujeres, con un $94 \%$ correspondiente al rango etario entre 60 y 74 años y un $6 \%$ al rango entre 75 y 89 años, se identificaron las enfermedades crónicas cardiovasculares y osteomusculares con el mayor porcentaje, siendo muy poco significativo el porcentaje de diabetes. Datos coincidentes con los informes epidemiológicos del último censo nacional 2010. 


\section{Alteraciones osteo-musculares}

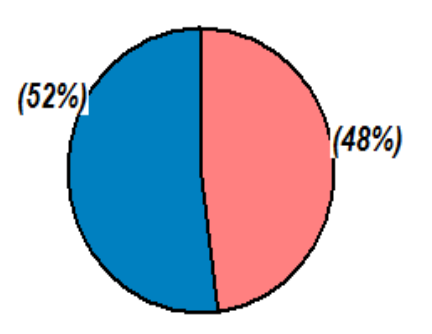

Disfunción Cardiovascular
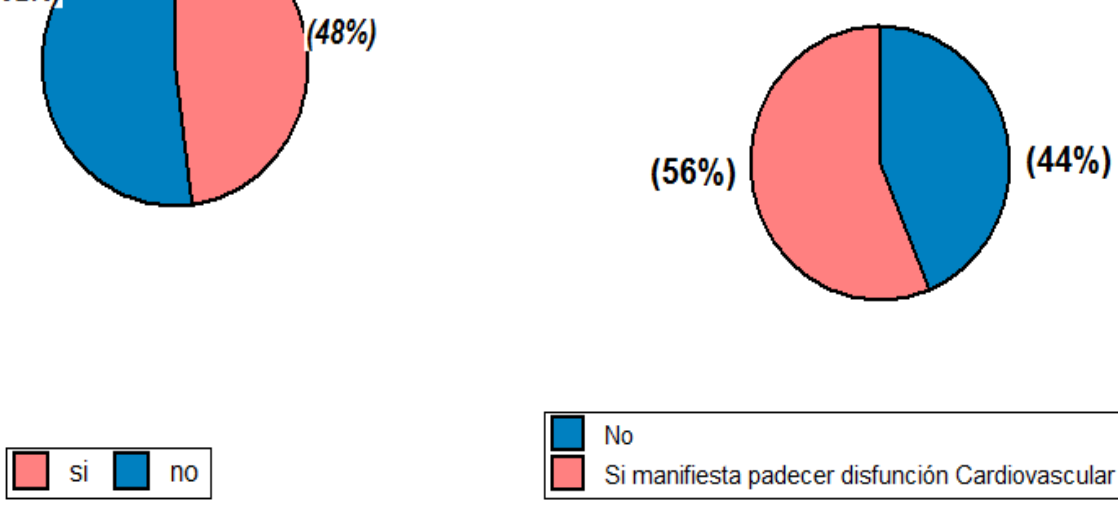

Si manifiesta padecer disfunción Cardiovascular

Fuente: Proyecto Investigación UARG.UNPA

\section{Diabetes}

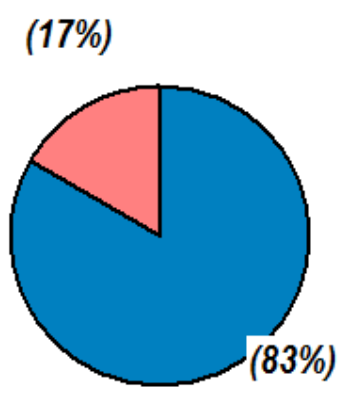

No $\quad \square$ Si manifiesta padecer Diabetes

Fuente: Proyecto Investigación UARG.UNPA

\subsection{Envejecimiento, Percepción de salud y Polifarmacia}

Considerando que la percepción es la imagen mental que se forma con la ayuda de las experiencias y motivaciones, la auto-percepción en salud constituye un indicador importante para conocer el estado de salud de los AM. Es clave por su capacidad predictiva sobre la morbilidad, mortalidad, calidad de vida y el aumento de la esperanza de vida. Así también para el nivel de bienestar a lo largo del ciclo vital, entendido éste como el grado de satisfacción que se tiene con el estado de salud física, mental y social.

La percepción de la salud está asociada a factores sociales, históricos-culturales, individuales y colectivos del proceso salud-enfermedad, a la morbilidad crónica y a la polimedicación. Entre las variables más fuertemente asociadas a la mala percepción de salud se encuentran la 
morbilidad crónica o aguda y de funcionamiento; así como también está asociada a variables psicológicas como la autoestima y la satisfacción con la vida,

El perfil epidemiológico de los AM en la Argentina muestra que las enfermedades crónicas prevalecen en forma epidémica y entre las más frecuentes figuran las alteraciones cardiovasculares, cáncer, diabetes, artrosis y la polifarmacia uno de los gigantes de la geriatría

El envejecimiento trae consigo enfermedades crónicas y/o discapacitantes que podrían incidir en una percepción negativa de la salud, sin embargo, las actitudes, sensaciones y/o vivencias del $10 \%$ de las enfermeras jubiladas de Rio Gallegos indican que perciben su salud como excelente, un $79 \%$ como Muy buena y un $10 \%$ la percibe regular.

\section{Percepción del propio estado de salud}

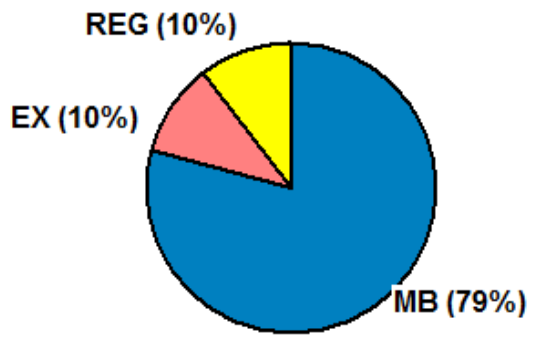

Fuente: Proyecto Investigación UARG.UNPA

Es interesante el dato que da cuenta que el $46 \%$ de ellas percibe que las enfermedades declaradas están asociadas al ejercicio de la profesión, siendo la de mayor asociación las artropatías /alteraciones musculo esqueléticas. 


\section{Alteraciones asociadas con el Ejercicio de la Profesión}

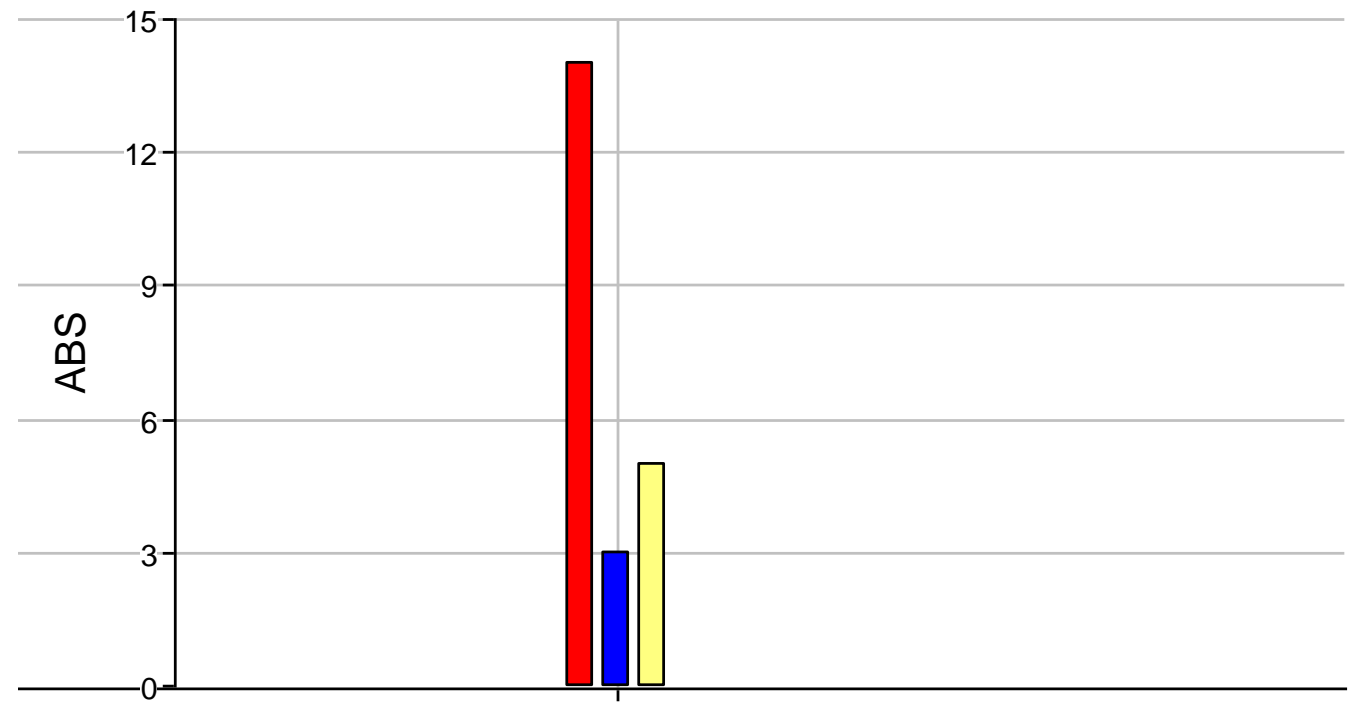

Artropatia

HTA

otro

Fuente: Proyecto Investigación UARG.UNPA

Azpiazu (2002) refiere que la percepción de salud es un constructo asociado a variables psicológicas como la autoestima y la satisfacción con la vida, que se ven influenciados por la salud mental y la capacidad funcional. Beaman, Reyes (2004) resaltan otros indicadores más objetivos como el número de enfermedades crónicas, el tiempo que han vivido con la enfermedad y la agudización de las mismas. Gallegos, Carrillo y otros, (2006) toman en cuenta varios factores sociales, de salud y organizacionales, y entre las variables más fuertemente asociadas a la mala percepción de salud se encuentran morbilidad crónica o aguda y de funcionamiento; por otra parte, Confortin, Giehl (2015) destaca la polimedicación con asociación significativa en la percepción de la salud.

Según la Organización Mundial de la Salud (OMS) en Hardon et al. (2004), el consumo de más de tres medicamentos simultáneos se define como polifarmacia. Algunos autores la indican entre 2 y 9 , siendo la más habitual de 5 fármacos; en este trabajo de investigación se adopto el criterio de la OMS de más de 3 fármacos considerando que tiene mayores ventajas preventivas. Así mismo la Organización Panamericana de la Salud (OPS, 2011) advierte que las personas mayores de 65 años tienen un promedio de consumo de 3 fármacos en los tratamientos ambulatorios, que aumenta a 7 fármacos durante las internaciones, y se calcula que por año se le prescriben entre 12 y 17 fármacos. Otro dato es que consumen el $25 \%$ de las prescripciones médicas.Datos de Argentina indican que entre el 65 a 94\% de los ancianos consume algún tipo de fármaco. En la población enfermera AM estudiada un porcentaje de 83 \%’ consume algún tipo de fármaco por día. 


\section{Consumo de Fármacos / dia}

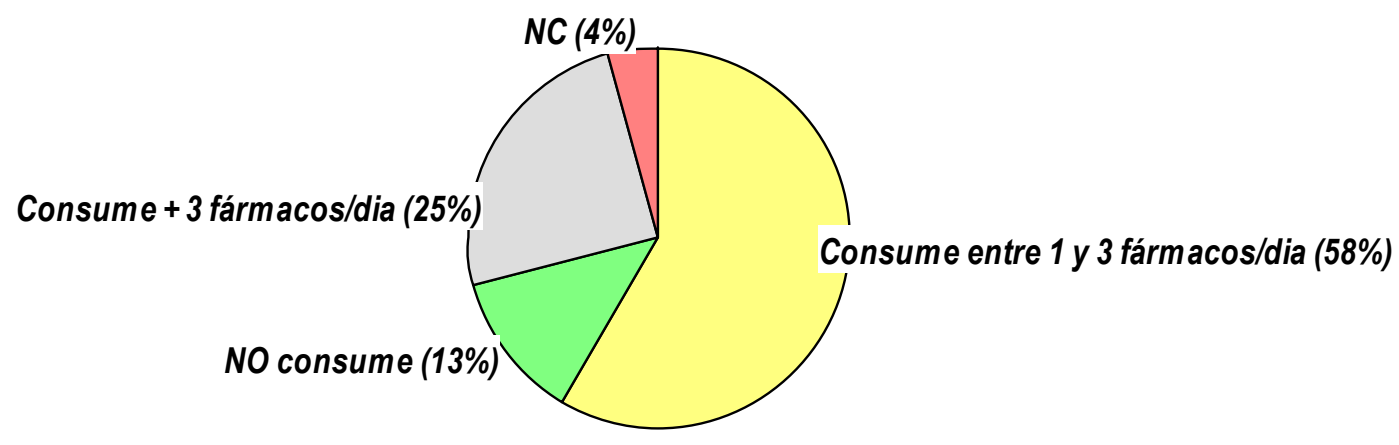

Fuente: Proyecto Investigación UARG.UNPA

En relación a la asociación de la polifarmacia con enfermedades crónicas resultó que el consumo de más de tres fármacos por día está asociado en un $100 \%$ al padecimiento de enfermedades crónicas.

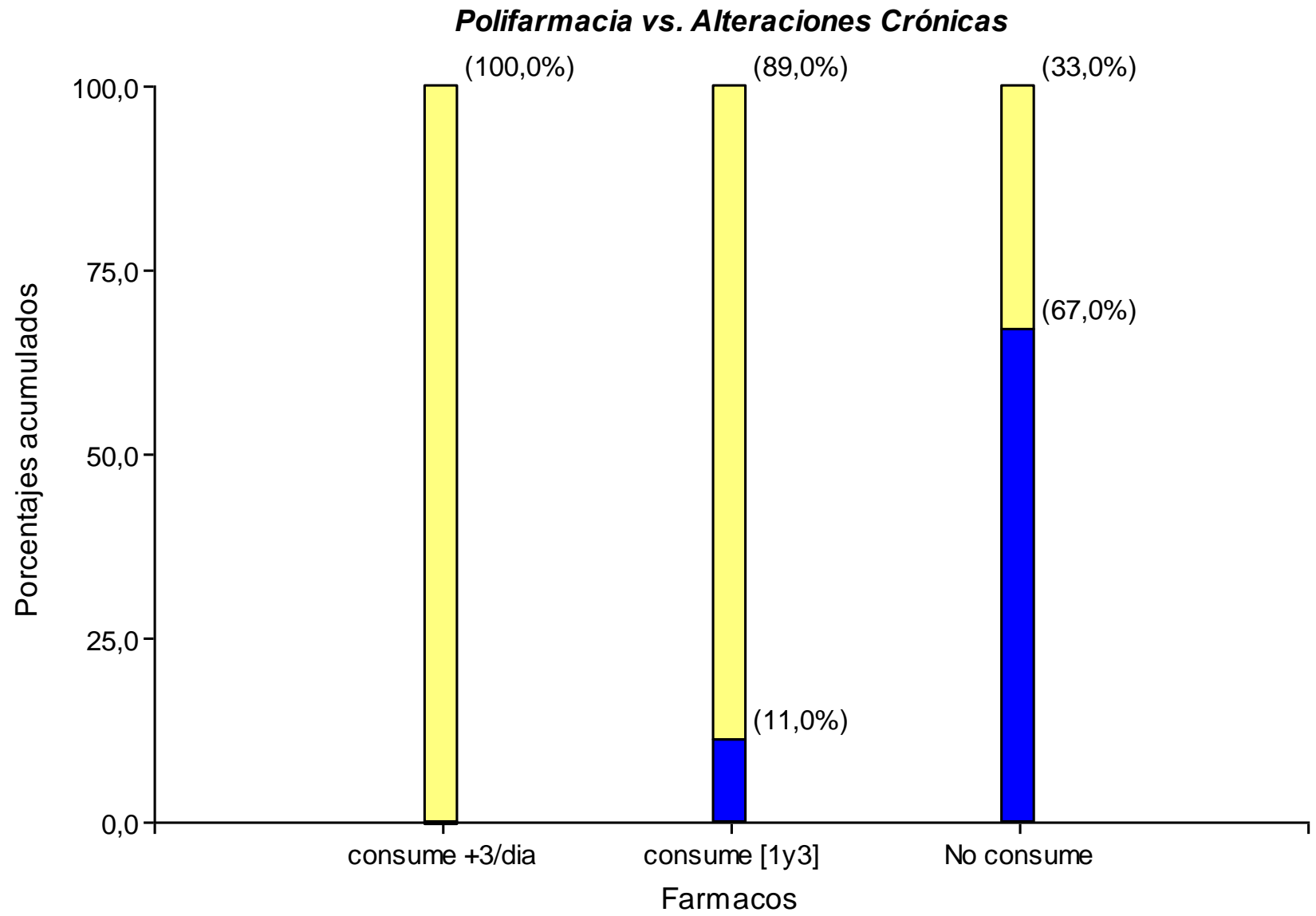

No padece alterac crónicas

Si padece alterac crónicas

Fuente: Proyecto Investigación UARG.UNPA 
Cuando se diferenció la polifarmacia en relación a las distintas patologías crónicas se encontró que para quienes padecían enfermedades musculo esqueléticas un $67 \%$ consumían más de tres fármacos /día, para las enfermedades cardiovasculares un $92 \%$ y para las metabólicas un $58 \%$.

El cruce de la variable Percepción de la salud y el consumo diario de fármacos mostró que el $100 \%$ de los individuos que dicen percibir su salud Regular, consumen alguna medicación cotidianamente y sólo un tercio (1/3) dice consumir entre uno y tres.

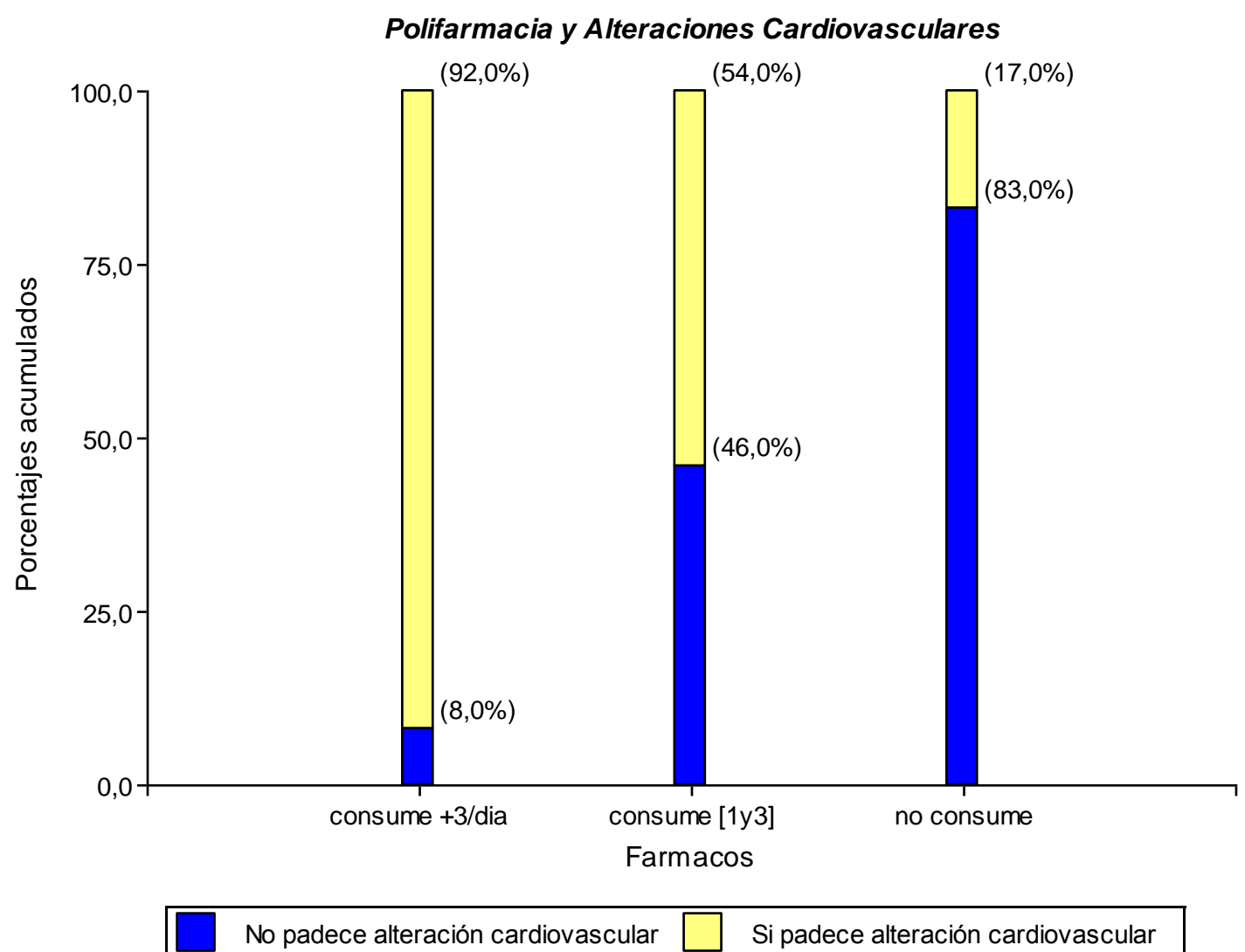

Fuente: Proyecto Investigación UARG.UNPA

Existe otra tendencia a definir la polifarmacia más desde el aspecto de medicación innecesaria que desde el número de fármacos, basada en registro de fármacos indicados a dosis subterapéuticas o que siguen indicados a pesar de no lograr su objetivo; es aquí donde se hace menester diferenciar entre la polifarmacia adecuada en la que el paciente ingiere varios medicamentos, todos con indicaciones correctas; de la polifarmacia inapropiada, en la que el paciente recibe más medicamentos que los indicados, y la pseudo polifarmacia redundante, en la que en la historia médica del paciente figura que tiene indicado más drogas de las que realmente consume (Rollason y Vogt, 2003). En esta investigación no se han obtenido datos respecto a esos aspectos de la polifarmacia. Si es válido destacar que un $94 \%$ de la población enfermera jubilada realizan controles médicos de salud y un $81 \%$ realiza tratamiento médico. 


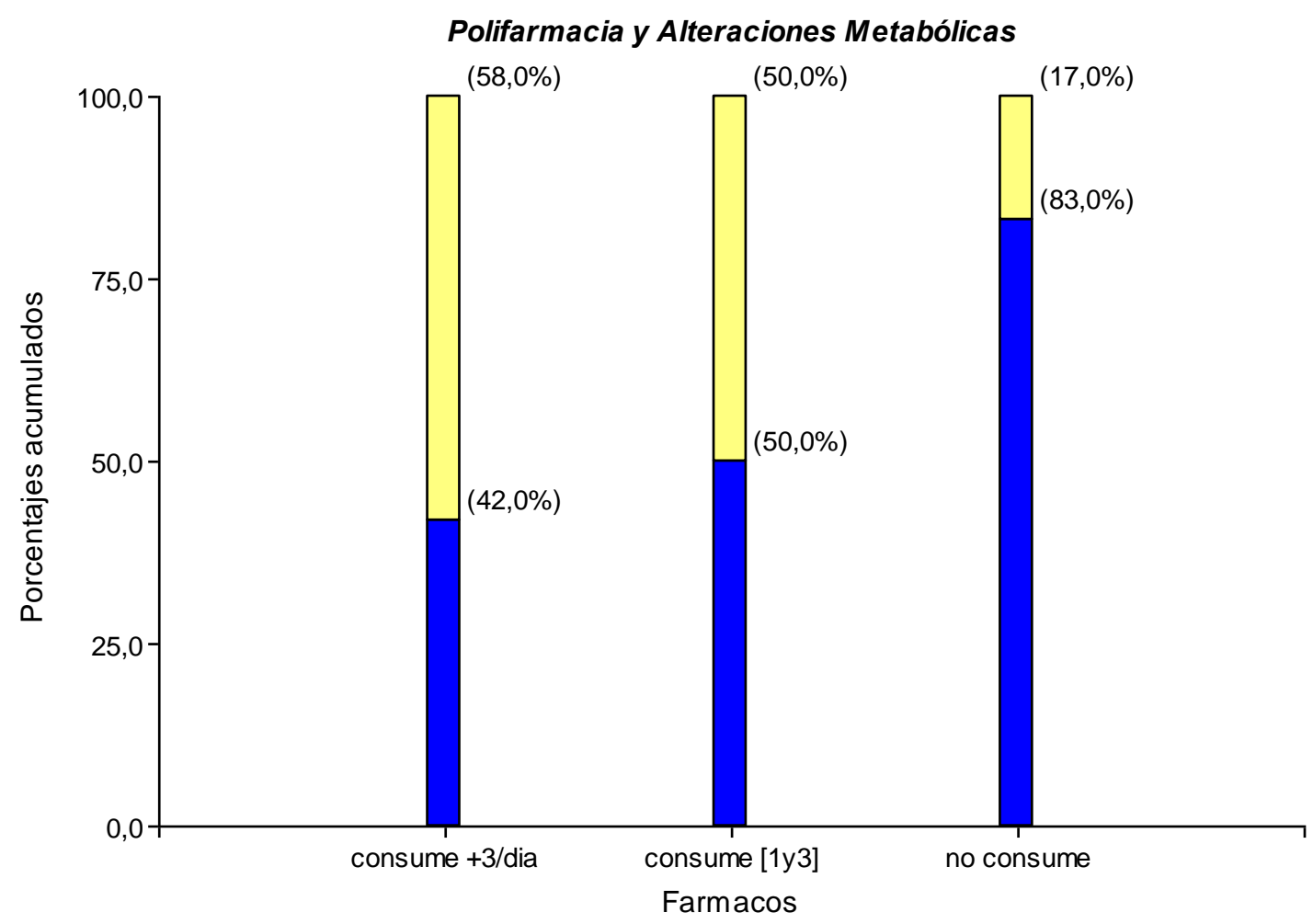

No padece Metabólica $\square$ Si padece Metabólica

Fuente: Proyecto Investigación UARG.UNPA

\section{Autopercepción de la Salud vs. Consumo de Fármacos}

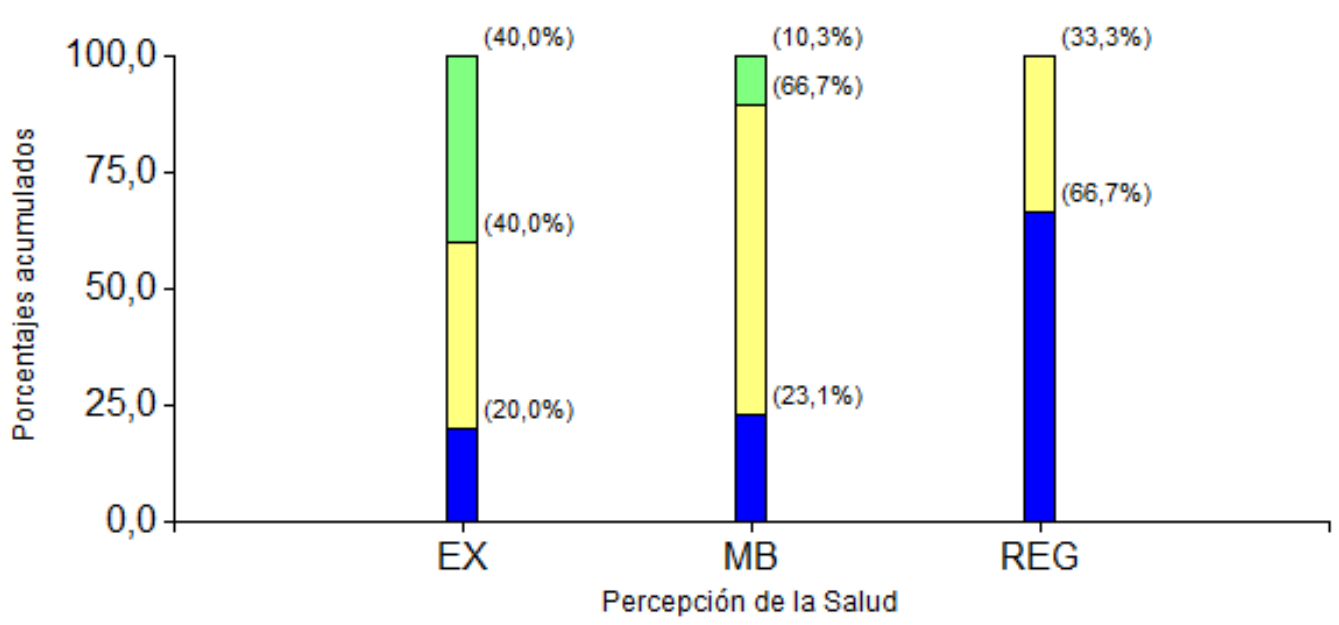

+3 fármacos /dia $\square$ [1 a 3] fármacos /dia $\square$ No consume

Fuente: Proyecto Investigación UARG.UNPA 


\section{Control Médico Periódico}

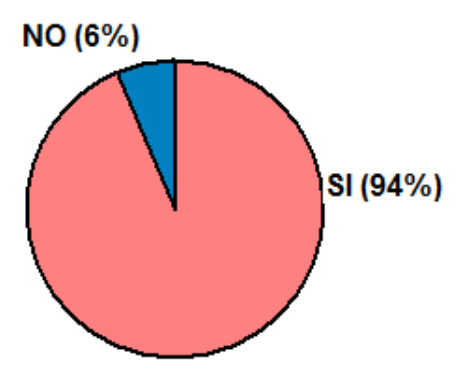

Fuente: Proyecto Investigación UARG.UNPA

Y entre el tratamiento médico y el consumo de más de tres fármacos resulto que el $100 \%$ de la población que los consumía lo hacía por prescripción médica.

\section{Consumo de fármacos vs. Tratamiento médico}

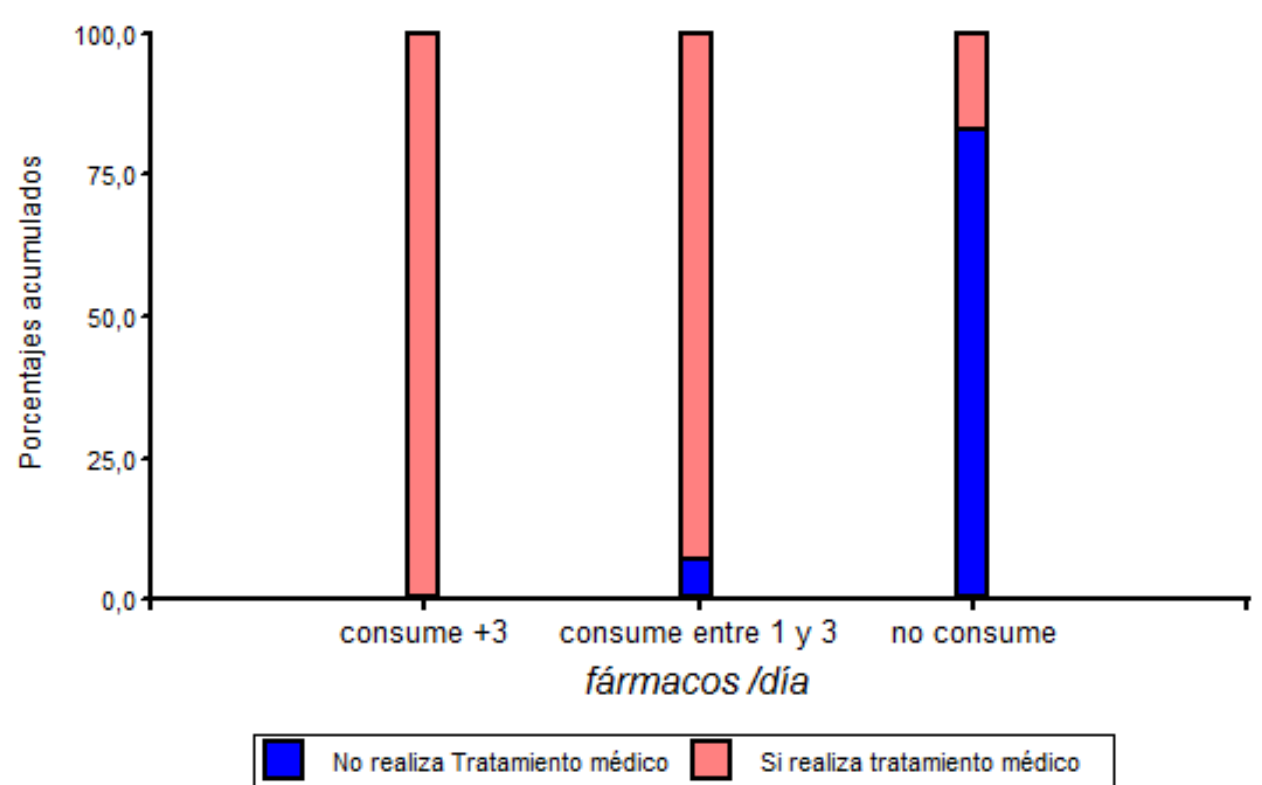

Fuente: Proyecto Investigación UARG.UNPA

El uso de fármacos representa un riesgo para la salud de los AM y un reto para la Salud Publica. Es importante destacar que confluyen varios factores que hacen difícil el diagnostico y la terapéutica, ya que el proceso de envejecimiento implica cambios fisiológicos como la disminución visual, auditiva, cambios cognitivos que pueden llevar a errores en el cumplimiento y adhesión al tratamiento; así mismo los cambios farmacocinéticos y fármacodinámicos que disminuyen la difusión, distribución y la eliminación de los fármacos, 
la mayor prevalencia de enfermedades crónicas, la polifarmacia y la automedicación aumentan el riesgo de interacciones y reacciones medicamentosas adversas. Los datos para Argentina indican que las reacciones secundarias a fármacos ocurren en el $25 \%$ de las personas mayores de 80 años, de las cuales el $20 \%$ son graves y que del 3 al $10 \%$ de las internaciones se deben a reacciones secundarias a fármacos, y un tercio de ellos son ancianos. Este factor no fue estudiado en esta investigación.

Por otra parte existen otros factores de riesgo significativos para la polifarmacia además de las polipatologias, tales como la visita a varios médicos por el mismo problema de salud, la automedicación, incluyendo entre ellas las terapias complementarias y/o alternativas que suelen no ser declaradas, factores que no fueron identificados en la población en estudio. Lo más importante a tener en cuenta es que la polifarmacia puede traer consecuencias físicas, económicas y sociales.

\section{CONCLUSIONES}

El perfil epidemiológico estudia la morbilidad y mortalidad de una determinada población indicando su estado de salud, en este artículo se identifican las principales alteraciones que manifestaron las enfermeras adultas mayores ubicándose en primer lugar las alteraciones cardiovasculares, seguida de las osteomusculares y la diabetes. Cuando se las relaciona con el ejercicio de la profesión se ubican en primer lugar a las artropatías, seguida de otras alteraciones entre las que se incluyen las metabólicas y las cardiovasculares en tercer lugar

En la relación con el consumo de más de tres fármacos, cambia el orden ubicándose en primer lugar las enfermedades cardiovasculares dando cuenta de la polifarmacia con esta alteración.

Es importante destacar que la farmacovigilancia y la intervención temprana de la Atención Primaria de la Salud (APS) son relevantes para evaluar las necesidades de apoyo y de aprendizaje de los AM con el fin de generar estrategias que garanticen la seguridad en el uso racional de los fármacos, no fue necesario realizar intervenciones en esta población.

Los datos muestran un estilo saludable en el manejo y administración de los fármacos en relación a enfermedades crónicas y su respectiva prescripción y seguimiento médico. Queda marcada la diferencia entre polifarmacia adecuada de la inapropiada, por lo que podría inferirse que el conocimiento incorporado durante años en su ejercicio profesional fue tomado como oportunidad en pos de buenas prácticas de salud en lo que a polimedicación se refiere.

Respecto a la percepción del estado de salud, un alto porcentaje (79 \%) de estas enfermeras AM perciben su salud como muy buena y cuando se relaciona esa autopercepcion con la polifarmacia, un $20 \%$ la percibe como excelente; datos significativos si se considera que la buena percepción de la salud incide en el bienestar fin último del desarrollo humano.

El rápido envejecimiento poblacional se convierte en un reto para las políticas regionales de salud pública y desarrollo social que deben dar respuesta a la crisis del cuidado que este cambio demográfico está anunciando en la Patagonia. Fortalecer e incorporar profesionales capacitados en gerontología es una de las medidas para garantizar esa calidad del cuidado. 


\section{BIBLIOGRAFIA}

ALVARADO OROZCO M., MENDOZA NÚÑEZ V. (2006). Prevalencia y factores de riesgo para polifarmacia en adultos mayores del Valle del Mezquital, Hidalgo. Revista mexicana de ciencias Farmacéuticas Vol. 37.Numero 4 Oct-Dic.

AZPIAZU M., JENTOFF A., VILLAGRASA J., ABANDES J., GARCIA N. \& ALVEAR F. (2002). Factores asociados a mal estado de salud percibido o mala calidad de vida en personas mayores de 65 años. Revista española de salud pública 76. 683-699.

BARRAGAN H., MOISO A., MESTORINO M., OJEA O., BARRAGAN S. (2007). "Fundamentos de salud pública". Perfil epidemiológico en Argentina.Edit Universidad La Plata. 2007. Cap 7 Pg 191-194.

BEAMAN R. (2004). Bienestar psicológico Apoyo social percibido y percepción de salud en Adultos Mayores, Terapia Psicológica Vol. 30 N².23-29.Sociedad Chilena de Psicología.

CEPAL (2010). Envejecimiento en América Latina: sistemas de pensiones y protección social integral.

CERVANTES BECERRA R., VILLARREAL RÍOS E., GALICIA RODRÍGUEZ L., VARGAS DAZZA E., MARTINEZ -GONZÁLEZ L. (2015). Estado de salud en el adulto mayor en atención primaria a partir de una valoración geriátrica integral. Atención Primaria 47 (6) 329-335.

CARRILLO R. Dr. "Plan Sintético de Salud Pública 1952 - 1958". Información Parlamentaria Honorable Cámara de Diputados de la Nación. Disponible en: https://www.ospat.com.ar/blog/salud/ramon-carrillo-politica-sanitaria-argentina/

CONFORTIN S., GIEHL M., LEDUR ANTES D., CEOLA SCHNEIDER I., D'ORSI E. (2015). Autopercepción positiva de salud en ancianos: estudio poblacional en el Sur de Brasil Saúde Pública, Rio de Janeiro, 31(5):1049-1060.

FASIO A., ROQUE M. (2008). Módulo 4 Salud, Epidemiologia y Envejecimiento Especialización en Gerontología comunitaria e institucional. Facultad de Psicología. Universidad Nacional de Mar del Plata. Ministerio de Desarrollo Social. Presidencia de la Nación.

FRENK J. (2009). "La salud de la población. Hacia una nueva salud pública". Fondo de Cultura Económica. México 1994.Editorial “EDIMED”, Buenos Aires, Argentina.

GONZÁLEZ GARCÍA G., TOBAR (2004). Salud para los argentinos economía, política y reforma del sistema de salud en Argentina. Editorial Granica S.A. Buenos Aires.

GALLEGOS-CARRILLO K., GARCÍA-PEÑA C., DURAN-MUÑOZ C., REYES H., DURAN-ARENAS L. (2006). Auto percepción del estado de salud: Una aproximación a los ancianos en México. Revista de Salud Pública 40 (5):792-801.

HARDON A., HODGKIN C. y DAPHNE F. (2004). Cómo investigar el uso de medicamentos por parte de los consumidores. OMS/ Universidad de Amsterdam.

INDEC (2010). Censo Nacional Argentina. Disponible en https://www.indec.gob.ar/

KAPLAN R., JAUREGUI J. R., RUBIN R. K. (2009). Los Grandes Síndromes Geriátricos. Edimed-Ediciones Médicas SRL, Buenos Aires. ISBN 978-987-24275-8-0.

LACUB R. (2014). Identidad y Envejecimiento. Perspectivas de occidente. Bs As, Paidos

LALONDE M. (1974). A new perspective on the healthof canadians. Disponible en: https://www.phac-aspc.gc.ca/ph-sp/pdf/perspect-eng.pdf

LAREDO L. M., VARGAS E., y MORENO A. (2005). Utilización de fármacos en geriatría. En: Velázquez. Farmacología Básica y Clínica. 17ª edición. Editorial Médica Panamericana S.A, p.1115-1119.

MARRINER TOMEY A., ALLIGOOD (2007). M. Modelos y Teorías de Enfermería. Elsevier $6^{\circ}$ Edición Madrid. 
MELLA R., GONZÁLEZ, D'APPOLONIO J., MALDONADO I., FUENZALIDA A. \& DIAZ A. (2004). Factores asociados al bienestar subjetivo en el adulto mayor, Psykhe 13. 79-89.

NOVOA GÓMEZ M. et al. (2005). Relación entre perfil psicológico, calidad de vida y estrés asistencial en personal de enfermería. Universitas Psychologica, 4(1), 63-76. http://www.scielo.org.co/scielo.php?script=sci_arttext\&pid=S1657-926720050001000 09\&lng=en\&tlng=en

ODDONE M. J. (1999). Envejecimiento y cambio social en "hechos y derechos", publicación n6, Subsecretaría de Derechos Humanos y Sociales, Buenos Aires, Argentina

OMS - Citado en British Medical Journal. (2000). 320: 237, 243. www.who.org

OSCANOA T. J. (2011). Diagnóstico de problemas relacionados con medicamentos en adultos mayores al momento de ser hospitalizados. Revista Perú Med. Exp Salud Pública. 28. 256-263

PELÁEZ E., ACOSTA D. L., CARRIZO E. (2015). Factores asociados al auto percepción de salud en adultos mayores. Revista cubano de Salud Pública. 41 (4) 638-648. https://scielo.sld.cu

PELÁEZ E., ACOSTA D. L., \& CARRIZO D. E. (2015). Factores asociados a la autopercepción de salud en adultos mayores. Revista Cubana de Salud Pública, 41(4) http://scielo.sld.cu/scielo.php?script=sci_arttext\&pid=S086434662015000400007\&lng =es\&tlng=es .

QUINTERO O. (compiladora).Organización Panamericana de la Salud. (2011). La salud de los adultos mayores: una visión compartida. Washington, D.C.: OPS, $2^{\mathrm{a}}$ Edición. Malciara

REGUEIRA NARANJO J., CONDE MARTIN M., BARRIO TAUPIER I., CERVERA ESTRADA L. (2000). Polifarmacia en la tercera edad. Revista Cubana Med. Gen Integ 16. 346-349.

ROLLASON y VOGT. (2003). Reducción de polifarmacia en el anciano: una revisión sistemática del papel del farmacéutico. Disponible en https://www.ncbi.nlm. nih.gov/pubmed/12964888 